\title{
SR instrumentation for optimized anomalous scattering and high resolution structure studies of proteins and nucleic acids (invited)
}

\author{
A. Deacon, J. Habash, S. J. Harrop, J. R. Helliwell, ${ }^{\text {a) }}$ W. N. Hunter, G. A. Leonard, and M. \\ Peterson \\ Department of Chemistry, University of Manchester, M13 9PL, United Kingdom
}

A. Hadener

University of Basle, Switzerland

A. J. Kalb (Gilboa)

Weizmann Institute, Rehovot, Israel

N. M. Allinson, C. Castelli, and K. Moon

Department of Electronics, University of York, Y01 SDD, United Kingdom

S. McSweeney

DRAL, Daresbury, WA4 4AD, United Kingdom

A. Gonzalez and A. W. Thompson

EMBL/ESRF, Avenue des Martyrs, Grenoble Cedex, France

S. Ealick, D. M. Szebenyi, and R. Walter

CHESS/MACCHESS, Cornell University, Ithaca, New York

(Presented on 18 July 1994)

\begin{abstract}
Crystal structure solution by anomalous dispersion methods has been greatly facilitated using the rapidly tunable station 9.5 at the Daresbury SRS. Both SIROAS and MAD techniques, with IP data, have been used in the phasing of a brominated nucleotide and a seleno deaminase, respectively. The electron density maps in each case are interpretable. Throughput of projects could be improved upon with a better duty cycle detector. Another category of data collection is that at very high resolution. Detailed structure refinement pushes the limits of resolution and data quality. Station 9.5 has been used to collect high resolution $(1.4 \AA)$ native data for the protein concanavalin A. This utilized very short wavelengths $(0.7 \AA)$, the image plate, and crystal freezing. A total of 155407 measurements from two crystals benefited from the on-line nature of the IP detector device, but a slow and quick pass are required to capture the full dynamic range of the data. There are data seen to $1.2 \AA$ and beyond for a pure Mn substituted form of the protein, but a higher intensity still is required to actually record these data. By comparison, trials at CHESS, on a multipole wiggler (station A1) with a CCD (without image intensifier) system, yield native concanavalin A data to $0.98 \AA$ and beyond. This demonstrates that the combination of yet higher intensity and the ease of use of a CCD offers worthwhile improvements; in this case an increase in the data by a factor of $(1.4 / 0.98)^{3}$, thus at least doubling the data to parameter ratio for protein structure model refinement and potentially opening up direct structure determination of proteins of the size of concanavalin A (25 kDa). Finally, possibilities at ESRF and further detector developments, such as mosaic CCDs and scintillator coatings, offer further impetus for the field. These include more intense rapidly tunable beams for anomalous dispersion-based structure solution and "ideal" higher resolution data collection and reactivity studies. ESRF BL19 is described; facilities on BL19 will include a system for freezing and storing crystals at cryogenic temperatures, so that data can be recorded from the same crystal on different runs. Overall, there have been tremendous strides made in this field in the last 15 years, and yet further improvements are to come. (C) 1995 American Institute of Physics.
\end{abstract}

\section{INTRODUCTION}

Synchrotron radiation is now used extensively in macromolecular crystallography. ${ }^{1}$ Instrumentation and source developments continue to be very important in all categories of data collection. The emittances of sources have improved considerably over the years, and come to better match the needs set by the sample acceptance. Hence, focusing of the beam to a size equal to the crystal sample cross section,

a) To whom correspondence should be addressed. within the constraints of keeping small convergence angles ( $<1 \mathrm{mrad}$ ), has become easier. This has allowed the setting up of instruments with the focusing and wavelength tuning functions separated. Optimized anomalous scattering data for structure solution, as well as high resolution data for protein structure refinement have, over the years, greatly facilitated protein structure analysis. (See for example Refs. 2-10.)

At Daresbury, the introduction of the high brightness lattice in 1985 was a major step in the SRS development. Station 9.5 at Daresbury exploits this, and has a design allowing a rapidly tunable setting of the wavelength for multiple 
wavelength anomalous dispersion applications, referred to as MAD. ${ }^{7}$ Moreover, it is also easier now to choose the wavelength for regular data collection; for very high resolution the selection of very short wavelengths (e.g., $0.5 \AA$ or shorter) is of interest. ${ }^{1}$

The ESRF offers further.improvements in source emittance and greater team intensity at short wavelengths. Hence, smaller samples will be accessible to study (e.g., for MAD), data can be recorded quicker and higher resolution data measured. At the ESRF, BL19 is being developed so as to serve optimized anomalous scattering applications in protein crystallography. There is now a large scale interest in the community for performing these experiments.

Detector development is gathering pace, as seen with the replacement of film by intensified TV detectors, MWPCs and the image plate. As exposure times are getting shorter, the potential exists for increasing the throughput of projects and improving the convenience of use. The duty cycle of the available devices is an increasingly critical parameter. Also, since multiple passes of data are required at different wavelengths for $M A D$, it is important that the detector medium and readout system allows the actual dynamic intensity range of the data to be collected in one sweep of the crystal if possible. These data sets, and those for very high resolution, are voluminous and the detector system must handle this problem. This is greatly assisted by having on-line readout. Also, as experiments become more ambitious, better data accuracy is required. CCDs represent a very promising line of development and are now being used in earnest at CHESS. Essentially they possess the advantages of the IP over film, without the poor duty cycle. There are also improvements in area coverage. Huge quantities of very accurate data can be measured with a CCD system both rapidly and conveniently.

This paper draws together various source and instrumentation developments for data collection in protein and nucleic acid crystallography. This is done with reference to a MAD study of a seleno deaminase, a SIROAS study of a brominated nucleotide, and very high resolution studies $(1.4 \AA$, $0.98 \AA$ ) of concanavalin A.

\section{SRS STATION 9.5}

The station design ${ }^{11}$ consists of a channel cut monochromator and a toroid mirror fed by the $5 \mathrm{~T}$ wiggler at the Daresbury SRS. The mirror is made of fused quartz coated with platinum, and set at an angle of $3 \mathrm{mrad}$ to reflect wavelengths greater than $0.5 \AA$. The wavelength can be rapidly tuned at an absorption edge. Over the small $\lambda$ range needed for MAD work, the vertical beam position does not move significantly for the narrow gap monochromator used, which is of the Hart ${ }^{12}$ design. The station design therefore extends the capability at the SRS, over the mirror plus triangular monochromator design of the earlier stations 7.2 and 9.6, which although more intense than 9.5 , are not so readily tunable. ${ }^{13,14}$ The commissioning of 9.5 was described at SRI91. ${ }^{15}$ Since then an on line image plate device (the MarResearch scanner) has been added, to replace film as the detector. This then represents the mode of operation used in the applications and results presented in Secs. III and IV A of this paper. There are recent improvements made to station 9.5 , involving a source position feedback control system, the incorporation of a kappa goniostat for sample alignment and oblique cut monochromator crystals for increasing the beam strength at $0.9 \AA$ for routine applications, which are described in Ref. 16. It should be noted that the instrument also readily allows removal of the monochromator to give a point focussed white beam for rapid Laue diffraction exposures. This is important for reactivity studies in protein crystals (see Refs. 17 and 18). For details of the Laue method, see Refs. 19 and 20 or monochromatic alternatives (e.g., Refs. 21 and 22) or a strategy based on a combination of both see Ref. 23. An application of the Laue mode of station 9.5 to myoglobin is described in Ref. 24. The station also lends itself to performing experimental data collection in the field of chemical crystallography with synchrotron radiation. ${ }^{25}$ This paper now concentrates on the results in two categories of data collection. These are anomalous dispersion applications and very high resolution data collection from protein crystals.

\section{RESULTS FROM STATION 9.5}

\section{A. MAD crystal structure study of a seleno met protein}

Hydroxymethylbilane synthase (HMBS), otherwise known as porphobilinogen deaminase, is a polymerase that catalyzes the conversion of porphobilinogen to hydroxymethylbilane. This is one step in a series of reactions that leads to the synthesis of haems, chlorophylls, or vitamin B12. The enzyme is about $40 \mathrm{KDa}$ molecular weight and contains six methionines. A selenomet variant has been produced and crystallized. ${ }^{26}$ The crystals are orthorhombic, space group $P 2{ }_{1} 22_{1}$ with unit cell dimensions of $a=88.4$, $b=76.4, c=50.6 \AA$. There is a single molecule in the asymmetric unit. The crystals are well-ordered and diffract beyond $2.5 \AA$ resolution. A MAD experiment has been carried out utilizing three wavelengths with a single crystal. The wavelengths were selected on the basis of an experimentally determined $\mathrm{x}$-ray fluorescence scan so as to minimize $f^{\prime}$ $(0.9794 \AA)$, maximize $f^{\prime \prime}(0.9789 \AA)$ and to have a reference wavelength where $f^{\prime}$ is near to zero $(0.9500 \AA)$. Crystallographic data were collected in angular wedges of $2.0 \mathrm{deg}$ each to minimize spot overlaps. Two wedges were collected contiguously at one wavelength so that the expected anomalous differences could be measured closely together in time. The crystal had been closely aligned so that the $f^{\prime \prime}$ differences were usually stimulated within each image. Another set of data at one wavelength was collected with the crystal not aligned in this way to facilitate scaling of data from one image to another in a different manner to the well aligned crystal data sets. The exposure time per image was typically $40 \mathrm{~s}$ (i.e., $20 \mathrm{~s}$ per deg). The readout time per image was 90 s. MAD phases to $3.0 \AA$ resolution were calculated with the program MLPHARE (i.e., treating the $f^{\prime}$ dip data set as the native). These phases produced an electron density map of good quality. The crystal structure of the wild-type HMBS has recently been reported; ${ }^{27}$ this structure was solved by MIR methods and thus provides the opportunity for a direct comparison of the MAD and MIR methods. A detailed account of this work will be presented later. Station 9.5 has 
also been used to perform a MAD experiment on a uranyl form of the protein Opp $\mathrm{A}^{28}$ and a brominated nucleic acid crystal. $^{29}$

\section{B. SIROAS and a brominated nucleotide heptamer}

This heptamer had a sequence d(GCATGCT), which was expected to adopt an unusual loop structure. Without a suitable search model, molecular replacement calculations failed. Access to a brominated derivative allowed a single isomorphous replacement with optimized anomalous scattering (SIROAS) type experiment ${ }^{30}$ to obtain phases. To optimize the anomalous signal of the bromine, data were measured at a wavelength of $0.91 \AA$ on station 9.5. Native data had been previously measured at $\mathrm{Cu} K \alpha$ on a Syntex P21 diffractometer. The crystals are orthorhombic, C222, with unit cell dimensions of $a=22.4, b=59.6, c=24.3 \AA$. The bromine atom position was readily interpreted from both the isomorphous and anomalous difference Pattersons. Following heavy atom refinement, phases were calculated using MLPHARE (figure of merit 0.74 ). The electron density map allowed a partial model to be constructed, from which an improved map could be calculated and a full model obtained. The structure was refined using XPLOR and NUCLSQ to 1.8 A. The asymmetric unit consists of a single strand of DNA which folds back on itself to form a novel loop structure for DNA. Two of these strands associate in an antiparallel manner, at approximately right angles to one another, forming a mini quadruplex structure. Such nucleic acid base tetramers are implicated in chromosomal or retroviral packaging. Full details of this work will be presented later. ${ }^{31}$

\section{TOWARD THE MEASUREMENT OF IDEAL DATA FOR THE PROTEIN CONCANAVALIN A}

Ideal data can be defined as being as free as possible from random and systematic (absorption) error, and which is to as high a resolution as possible; the realization of this involving shorter wavelengths and high intensity using synchrotron radiation has been suggested ${ }^{32}$ and criticized. ${ }^{33}$ The application of this approach to the structural study of the archetypal lectin protein concanavalin $\mathrm{A}$ and its interactions with saccharides is of structural interest; earlier work has been at 2.0 and $1.6 \AA$ resolution for saccharide bound and saccharide-free forms of the protein. ${ }^{34,35}$ The finer details of the protein saccharide interactions will be elucidated by use of such "ideal" data.

\section{A. Very high resolution (1.4 $\AA$ ) native concanavalin A studies at the SRS with IP}

Station 9.5 was used to collect very high resolution data from the native concanavalin A protein. The wavelength used was $0.7 \AA$ so as to take advantage of the transparency of the crystal at shorter wavelengths and the more compressed data collection geometry (i.e., the spots strike the detector less obliquely than at longer wavelengths). Ideally a wavelength shorter than $0.7 \AA$ would be used (e.g., $0.5 \AA$ ) but the beam intensity on station 9.5 is fading here. Frozen crystals were used so as to prevent radiation damage to the sample during data collection, as is now quite a common practice in the field. In order to collect the full dynamic range of the reflection intensity data both a slow and a quick pass data set were required. The total angular sweeps were 60 and $135 \mathrm{deg}$; the angular ranges per image were $0.9 \mathrm{deg}$ and $1.4 \mathrm{deg}$; the exposure times per image were $\approx 200 \mathrm{~s}$ and $\approx 40 \mathrm{~s}$, respectively; the mosaic spreads were $0.65 \mathrm{deg}$ in each case. The $\mathrm{x}$-ray beam struck the center of the detector (i.e., symmetric setting), Fig. 1(a). The samples were randomly oriented. In all, 195 images were processed using the MOSFLM version 5.20 program (A. Leslie). The processing statistics are shown in Table I indicating data of very reasonable quality, high completeness, and good multiplicity. The limitations of the data collection were first, the duty cycle of the detector $(90 \mathrm{~s}$ readout) especially for the quick pass data set, and second the fading intensity of the beam for the slow pass data set, and which impacts on the overall resolution limit obtained for the data of $1.4 \AA$.

\section{B. Use of CHESS MW with Princeton $1 \mathrm{k}$ CCD detector ${ }^{36}$ to record $0.98 \AA$ resolution data}

Station $A 1$ at CHESS is fed by a 25 pole MW device. It has a focussed beam and a wavelength of $0.9 \AA$ (minimum possible at the time). The intensity at the sample position is $5 \times 10^{12} \mathrm{ph} / \mathrm{s} / \mathrm{mm}^{2} .^{37}$ Hence, the intensity is better than SRS $9.5\left(3.6 \times 10^{11}\right)$ but the choice of wavelength is not so flexible on $\mathrm{A} 1$.

By displacing the $50 \times 50 \mathrm{~mm}^{2}$ aperture CCD off from the main beam, so that the latter is in one corner of the detector aperture, a resolution limit of $1.1 \AA$ at the top and side of the detector is realized, and with $0.98 \AA$ into one corner. Indeed a frozen native concanavalin A crystal diffracted right to the corner of the detector [Fig. 1(b)]. A complete data set was recorded on three separate occasions from three crystals involving slow and quick passes, the latter to record overloaded spots. In all, 554 images of 0.5 deg each with $20 \mathrm{~s}$ per image (slow pass) and 67 images of $1.5 \mathrm{deg}$ each with $5 \mathrm{~s}$ per image (quick pass) were used. The dead time between images was $25 \mathrm{~s}$. The image data were corrected according to standard procedures ${ }^{36}$ and the data integrated and scaled using DENZO and SCALEPACK (Z. Otwinowski and W. Minor, Table II).

Comparing this run on A1 with CCD with that of SRS 9.5 with IP is clearly of interest. Basically the strong beam intensity on A1 makes the recording of data to $0.98 \AA$ (and beyond) possible. Also the duty cycle is more favorable with the CCD but the large area of the IP is a strength.

How far does this concanavalin A crystal form diffract? Very recent tests at $\mathrm{Al}$ with IP placed close to the crystal shows diffraction to $0.90 \AA$ and beyond. For a protein of this size, these resolutions are remarkable, although $0.96 \AA$ from frozen trypsin $(20 \mathrm{kDa})$ has been reported recently. ${ }^{38}$

To optimally measure concanavalin A data at such high resolutions as $0.85 \AA$ will benefit from fine phi slices (e.g., 0.2 deg or less) and also a wavelength such as $0.5 \AA$ where the geometry (especially obliquity) is more favorable, in addition to the better transparency of the sample. At CHESS, work is in progress to implement a mosaic CCD detector with a fine focus $0.5 \AA$ wavelength beam as an option. 

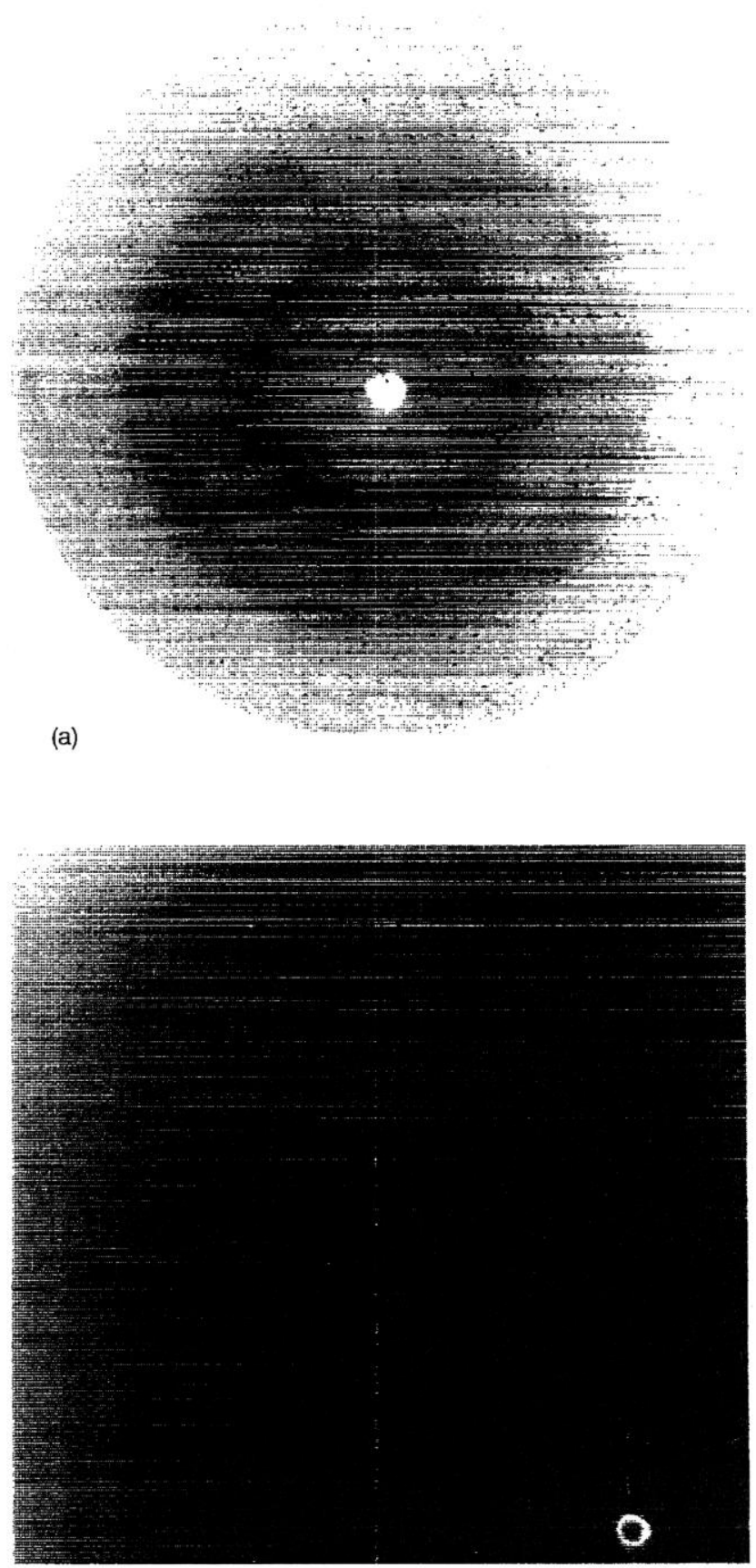

(b)

FIG. 1. Toward "ideal" data collection. Monochromatic oscillation images from frozen crystals of native concanavalin A: measured at (a) the SRS 3-pole wiggler with on-line IP as detecting medium showing data extending to $1.4 \AA$ resolution ( $0.9 \mathrm{deg}$ angular range). (b) CHESS 25 -pole wiggler with on-line CCD detector showing data extending to $0.98 \AA$ resolution in the top corner (0.5 deg angular range).

\section{DEVELOPMENT OF ESRF BL19 FOR MAD}

BL19 is being constructed on an ESRF bending magnet for $\mathrm{MAD},{ }^{39,40}$ using the hard $(0.85 \mathrm{~T})$ end of the bending magnet spectrum giving a critical wavelength of $0.6 \AA$, and a net, vertically integrated, power of $78 \mathrm{~W}$ per horizontal mrad. Up to 4 horizontal mrad are available, of which 2 will initially be used. The beam line optics consists of a double
TABLE I. SRS Station 9.5+ IP+ frozen native concanavalin A: (a) $R$ factors (I) vs resolution, D, up to $1.4 \AA$. (b) Data completeness and multiplicity vs resolution D, up to $1.4 \AA$ (both extracted from MOSFLM 5.20 output).

\begin{tabular}{|c|c|c|c|c|}
\hline$\langle D(\AA)\rangle$ & $\mathrm{R}$ fac $(\mathrm{I})^{\mathrm{a}}$ & $\mathrm{I}^{\mathrm{b}} / \mathrm{sigma}$ & $\mathrm{N}$ meas & $\mathrm{N}$ unique \\
\hline 4.43 & 0.052 & 8.6 & 9689 & 1560 \\
\hline 3.13 & 0.054 & 10.5 & 18100 & 2775 \\
\hline 2.56 & 0.069 & 8.8 & 23507 & 3544 \\
\hline 2.21 & 0.099 & 6.1 & 26992 & 4159 \\
\hline 1.98 & 0.110 & 5.5 & 23125 & 4308 \\
\hline 1.81 & 0.071 & 8.7 & 7528 & 2397 \\
\hline 1.67 & 0.092 & 7.5 & 6978 & 2403 \\
\hline 1.57 & 0.120 & 5.9 & 6393 & 2364 \\
\hline 1.48 & 0.161 & 4.5 & 5724 & 2211 \\
\hline 1.40 & 0.184 & 3.9 & 2779 & 1161 \\
\hline$\langle\mathrm{D}(\AA ̊)\rangle$ & $\mathrm{N}$ meas & $\mathrm{N}$ unique & Completeness (\%) & Multiplicity \\
\hline 4.43 & 9731 & 1572 & 98.5 & 6.2 \\
\hline 3.13 & 8163 & 2781 & 99.7 & 6.5 \\
\hline 2.56 & 23618 & 3553 & 100.0 & 6.6 \\
\hline 2.21 & 27129 & 4165 & 99.9 & 6.5 \\
\hline 1.98 & 24344 & 4639 & 98.7 & 5.2 \\
\hline 1.81 & 11350 & 4387 & 85.7 & 2.6 \\
\hline 1.67 & 11222 & 4657 & 83.9 & 2.4 \\
\hline 1.57 & 10996 & 4896 & 82.1 & 2.2 \\
\hline 1.48 & 10786 & 5100 & 80.7 & 2.1 \\
\hline 1.40 & 8068 & 4633 & 69.3 & 1.7 \\
\hline Totals & 155407 & 40383 & 86.8 & 3.8 \\
\hline
\end{tabular}

${ }^{a} \mathrm{R}$ factors (I) for the strongest reflection band and for the complete data set. I max 32412, R fac 0.064 TOTALS 0.071 .

${ }^{b}$ The partial bias on $\mathrm{I}$ is $5.3 \%$.

mirror arrangement sandwiching a channel cut monochromator (Fig. 2). The first mirror will collimate the beam in the vertical direction (during the initial operation it will act as a short wavelength filter without collimation), the second (a toroid) will perform the horizontal focusing. Both the mirrors and their bending mechanisms are currently being produced by Zeiss. The monochromator is a channel cut device for easy and rapid interchangeability. In this way, the net throughput bandpass (and hence the intensity on the sample) can be easily varied to match either a narrow bandpass MAD

TABLE II. CHESS station A1 + CCD + frozen native concanavalin A: $\mathrm{R}$ factors and completeness vs resolution (extracted from SCALEPACK output).

\begin{tabular}{lccc}
\hline \hline D range & N unique & Completeness $(\%)$ & $R$ factor (I) \\
\hline $99.0-2.11$ & 13946 & 97.6 & 0.047 \\
$2.11-1.68$ & 12627 & 90.5 & 0.073 \\
$1.68-1.46$ & 11488 & 82.6 & 0.079 \\
$1.46-1.33$ & 11152 & 80.9 & 0.088 \\
$1.33-1.23$ & 10898 & 79.0 & 0.091 \\
$1.23-1.16$ & 10487 & 76.3 & 0.095 \\
$1.16-1.10$ & 8495 & 61.6 & 0.084 \\
$1.10-1.06$ & 4358 & 31.8 & 0.081 \\
$1.06-1.02$ & 1982 & 14.4 & 0.111 \\
$1.02-0.98$ & 324 & 2.4 & 0.145 \\
Totals & 85757 & 62.0 & 0.055 \\
\hline \hline
\end{tabular}

Total number of measurements 290035 yielding 85757 unique (i.e., average multiplicity is 3.38 ).

${ }^{b}$ Average I/sig(I) was 16.7 .

'No reflections rejected. 


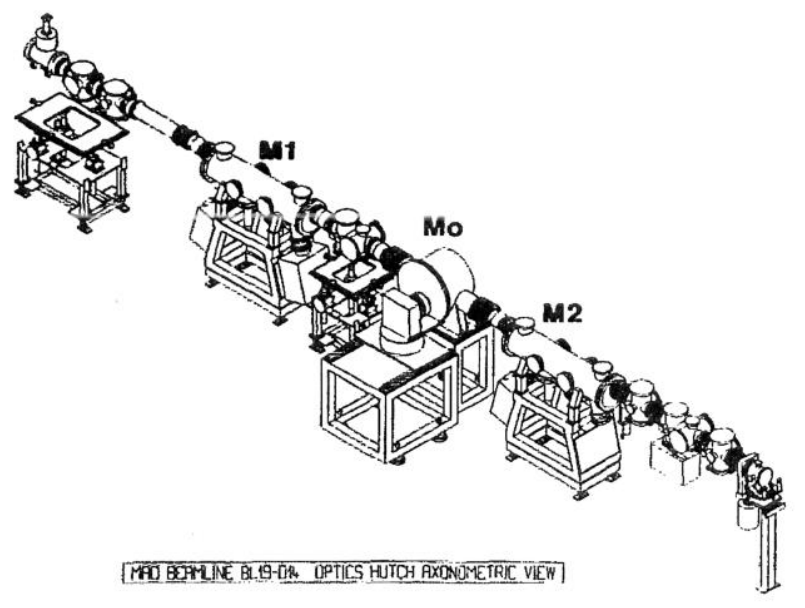

FIG. 2. Instrumentation for optimized anomalous scattering in protein crystallography. ESRF beamline 19 (BL19) optimized for MAD: layout of the optical scheme consisting of a collimating mirror, channel cut monochromator, and toroid focusing mirror.

application or a wider bandpass SIROAS experiment. Initially, only a $\mathrm{Si}(111)$ monochromator will be available, but both $\mathrm{Ge}$ and asymmetrically cut $\mathrm{Si}$ monochromators are under study. In this way, the bandpass may be selected according to the experimental requirement covering the approximate limits of $10^{-4}$ and $5 \times 10^{-5}$. The monochromator vessel will contain a selection of metal foils for rapid wavelength recalibration. Samples will be mounted on a Kappa goniostat with 2 detector arms. The first arm will carry a rapid carousel image plate changing system for six $20 \times 25 \mathrm{~cm}$ plates. The second will be available for a CCD detector. Image plates will be scanned initially by a commercial scanner, but eventually by a large area drum reader being developed at EMBL Grenoble. ${ }^{41}$ It is likely that many MAD data collection runs at the ESRF will involve smaller samples, thus increasing the need for radiation damage protection of the sample. Hence, cryo-temperatures will be an important feature of the MAD work. A system consisting of an Oxford Instruments Cryostream device, a device to rapidly transfer the sample to the beam and a frozen sample storage facility is being developed. The station is currently being constructed, and it is expected that it will be collecting data in commissioning mode in the spring of 1995.

\section{CCD DETECTOR DEVELOPMENTS}

CCDs have long been associated with detector developments. It was an obvious step to replace the TV camera tube in intensified area detector systems with a CCD imager. The CCD inherently provides better geometric precision, higher dynamic range, lower noise floor and much reduced interaction with external electromagnetic fields. This basic detector topology-namely, phosphor/pre-storage gain/optical coupler/CCD - has been refined to the stage where x-ray image intensifiers, with apertures greater than $200 \mathrm{~mm}$ diameter, optically coupled to slow-scan CCD cameras can provide high quality diffraction data. The developments at the ESRF are indicative of the present status of such systems, with detective quantum efficiencies (DQE) in excess of $40 \%$ over the energy range 5 to $25 \mathrm{keV}$ and a measured pointspread function (PSF) of about $700 \mu \mathrm{m}$ at $1 \%$ of peak intensity. ${ }^{42}$

The most critical element of any detector system is the primary converter. Though there is no fundamental difference in the physical processes involved in the wavelength conversion from $x$-ray photons to visible ones, it is useful to consider separately, phosphors as powder-based layers (usually floated on to their underlying substrates) and scintillators as vapor-deposited layers with some degree of crystal structure. There are a number of requirements for both phosphors and scintillators that must be met if they are to be successfully employed. The most important are a high conversion efficiency from $x$-ray photons to visible ones, and the preservation of a high spatial resolution. There are over a dozen inorganic materials that have been examined as possible $\mathrm{x}$-ray phosphors, with the most popular being $\mathrm{Gd}_{2} \mathrm{O}_{2} \mathrm{~S}: \mathrm{Tb}$ and $\mathrm{Y}_{2} \mathrm{O}_{2} \mathrm{~S}{ }^{43,44}$ The ability to achieve high conversion efficiencies allows the elimination of the prestorage gain element in the detector chain and still preserve a sufficiently high DQE for low integrated spot intensities. The use of structured scintillators as the primary converter is a very promising alternative, since not only can high conversion efficiencies be maintained but the PSF of the scintillator itself can be insignificant; the system's limiting PSF being determined by the dispersion in the optical components and the visible imaging performance of the $\mathrm{CCD}$. Structured CsI:Tl screens have been measured with a PSF as low as 200 $\mu \mathrm{m}$ diameter at the $1 \%$ of peak intensity. ${ }^{45}$ The importance of low PSF on DQE for low intensity spots cannot be overemphasized. ${ }^{46}$ As the total integrated intensity of a weak spot represents a small number of collected photons (if integration times are to be kept realistic) then any significant dispersion of these photons over the tessellated structure of the $\mathrm{CCD}$ array will result in the loss of detectable photoinduced charge. The reduction of the PSF is the most critical aspect of detector design for high resolution diffraction studies.

Omitting the pre-storage gain element (i.e., image intensifiers or microchannel plates) from the detector means that additional sources of noise (e.g., the equivalent background illumination) and dispersive factors (that adversely affect the PSF and overall geometric accuracy) are also eliminated. The optical coupling from the phosphor or scintillator screen to the $C C D$ is best performed using coherent optical fiber tapers rather than lenses, as the transmission efficiency of tapers is far superior for demagnifying optics. Even so, the transmission efficiency of tapers (and for that matter, lenses) falls dramatically with increasing demagnification ratios. In practical systems, the demagnification ratio is limited to $3: 1$ at most. As scientific-grade CCDs are typically between $1 \times 1$ $\mathrm{cm}^{2}$ and $3 \times 3 \mathrm{~cm}^{2}$, and the overall detector aperture is, at least, an order of magnitude greater, this implies the need to use a mosaic of fiber-coupled CCD modules. ${ }^{47}$ The use of a number of individual CCDs, each with 2 or 4 readout channels, can reduce the dead-time of the detector to a few tens of ms yet maintain a low video bandwidth for each individual channel (and so not compromise the excellent low-noise per- 
formance of CCDs). CCD systems offer a high degree of flexibility in their operation (e.g., variable integration and readout rates, on-chip pixel binning, and fast time-slicing, ${ }^{48}$ and this flexibility can be exploited in the effective collection of data for a particular experiment.

\section{ACKNOWLEDGMENTS}

We are very grateful to A. Liljas for his support of the station 9.5 development over a number of years and C. Nave for many useful discussions concerning station 9.5 and its development. We thank A. Battersby, T. L. Blundell, S. Wood, and G. Louie for their interest and support of the MAD experiment on Se HMBS. We thank C. Branden, S. Cusack, C. Wilkinson, and A. Kvick at ESRF/EMBL for their interest and support of the ESRF beamline 19 (BL19) development. At Cornell University and CHESS, P. Doing, Qun Shen, B. Miller, and J. Navaie are thanked for their help and support of the "ideal" data collection work and development. Data processing was done with MOSFLM 5.12 and 5.20 as well as DENZO and SCALEPACK; we are grateful to A. Leslie, Z. Otwinowski, and W. Minor for use of these programs. We are very grateful to EEV Ltd., Chelmsford, UK including in particular B. Allen, J. E. U. Ashton, I. Flint, and P. J. Pool for providing modified CCD detectors and great assistance over the years.

${ }^{1}$ J. R. Helliwell, Macromolecular Crystallography with Synchrotron Radiation (Cambridge University Press, Cambridge, 1992).

${ }^{2}$ J. C. Phillips, A. Wlodawer, M. M. Yevitz, and K. O. Hodgson, Proc. Natl. Acad. Sci. USA 73, 128 (1976).

${ }^{3}$ J. R. Helliwell, Proceedings of the Daresbury Study Weekend DL/SCI R13 1-6 (1979).

${ }^{4}$ N. Sakabe, K. Sakabe, and K. Sasaki, in Structural Studies of Biological Interest: A Volume in Honour of Dorothy Hodgkin, edited by G. Dodson, J. P. Glusker, and D. Sayre (Oxford University Press, Oxford, 1981).

${ }^{5}$ K. S. Wilson, E. A. Stura, D. L. Wild, R. J. Todd, D. I. Stuart, Y. S. Babu, J. A. Jenkins, T. S. Standing, L. N. Johnson, R. Fourme, R. Kahn, A. Gadet, K. S. Bartels, and H. D. Bartunik, J. Appl. Crystallogr. 16, 28 (1983).

${ }^{\circ}$ J. R. Helliwell, Rep. Prog. Phys. 47, 1403 (1984).

${ }^{7}$ W. Hendrickson, Trans. Am. Crystallogr. Assoc. 21, 11 (1985).

${ }^{8}$ R. Kahn, R. Fourme, R. Bosshard, M. Chiadmi, J. L. Risler, O. Dideberg, and J. P. Wery, FEBS Lett. 179, 133 (1985).

${ }^{9}$ J. M. Guss, E. A. Merritt, R. P. Phizackerley, B. Hedman, M. Murata, K. O. Hodgson, and H. C. Freeman, Science 241, 806 (1988).

${ }^{10}$ V. Ramakrishnan, J. Finch, V. Graziano, P. L. Lee, and R. Sweet, Nature 362, 219 (1993)

${ }^{11}$ R. Brammer, J. R. Helliwell, W. Lamb, A. Liljas, P. R. Moore, A. W. Thompson, and K. Rathbone, Nucl. Instrum. Methods A 271, 678 (1988). ${ }^{12}$ R. Cernik and M. Hart, Nucl. Instrum. Methods A 281, 403 (1989).

${ }^{13}$ J. R. Helliwell, T. J. Greenhough, P. D. Carr, S. A. Rule, P. R. Moore, A. W. Thompson, and J. S. Worgan, J. Phys. E 15, 1363 (1982).

${ }^{14}$ J. R. Helliwell, M. Z. Papiz, I. D. Glover, J. Habash, A. W. Thompson, P. R. Moore, N. Harris, D. Croft, and E. Pantos, Nucl. Instrum. Methods A 246, 617 (1986).

${ }^{15}$ A. W. Thompson, J. Habash, S. Harrop, J. R. Helliwell, C. Nave, P. Atkinson, S. S. Hasnain, I. D. Glover, P. R. Moore, N. Harris, S. Kinder, and S. Buffey Rev. Sci. Instrum. 63, 1062 (1992).

${ }^{16} \mathrm{~S}$. McSweeney and C. Nave, Rev. Sci. Instrum., these proceedings.
${ }^{17}$ H. D. Bartunik, E. Jerzembek, D. Pruss, G. Huber, and H. C. Watson, Acta Crystallogr. A 37 C 51 (1981).

${ }^{18}$ D. W. J. Cruickshank, J. R. Helliwell, and L. N. Johnson, (eds.) TimeResolved Macromolecular Crystallography (The Royal Society and OUP, 1992).

${ }^{19}$ K. Moffat, D. M. Szebenyi, and D. H. Bilderback Science 223, 1423 (1984).

${ }^{20}$ A. Cassetta, A. Deacon, C. Emmerich, J. Habash, J. R. Helliwell, S. McSweeney, E. Snell, A. W. Thompson, and S. Weisgerber, Proc. R. Soc. London Ser A 442, 177 (1993).

${ }^{21}$ N. Sakabe, Nucl. Instrum. Methods A 303, 448 (1991).

${ }^{2} \mathrm{~J}$. Hajdu and I. Andersson, Ann. Rev. Biophys. Biomol. Struct. 22, 467 (1993).

${ }^{23}$ S. Weisgerber and J. R. Helliwell, J. Chem. Soc. Faraday Trans. 89, 2667 (1993).

${ }^{24}$ A. Cameron, S. J. Smerdon, A. J. Wilkinson, J. Habash, J. R. Helliwell, Li Tiangsheng, and J. S. Olson, Biochem. 32, 13061 (1993).

${ }^{25}$ E. Snell, J. Habash, M. Helliwell, J. R. Helliwell, J. Raftery, V. Kaucic, and J. W. Campbell, J. Synchrotron. Rad. (in press).

${ }^{20}$ A. Hadener, P. K. Matzinger, V. N. Malashkevich, G. V. Louie, S. P. Wood, P. Oliver, P. R. Alefounder, A. R. Pitt, C. Abell, and A. R. Battersby, Eur. J. Biochem. 211, 615 (1993).

${ }^{27}$ G. V. Louie, P. D. Brownie, R. Lambert, J. B. Cooper, T. L. Blundell, S. P. Wood, M. J. Warren, S. C. Woodcock, and P. M. Jordon, Nature 359, 33 (1992).

${ }^{28}$ I. D. Glover, R. C. Denny, N. D. Ngutu, S. M. McSweeney, A. W. Thompson, E. J. Dodson, A. J. Wilkinson, and J. R. H. Tame, Acta D (in press).

${ }^{20} \mathrm{M}$. Peterson et al. (in preparation).

${ }^{30}$ P. Baker, G. W. Farrants, T. J. Stillman, K. L. Britton, J. R. Helliwell, and D. Rice, Acta Crystallogr. A 46, 721 (1991).

${ }^{31}$ G. Leonard, S. Zhang, M. Peterson, S. J. Harrop, J. R. Helliwell, W. B. Cruse, B. Langlois d'Estaintot, O. Kennard, T. Brown, and W. N. Hunter (in preparation).

${ }^{32}$ J. R. Helliwell, S. Ealick, P. Doing, T. Irving, and D. M. Szebenyi, Acta Crystallog. D 49, 120 (1993).

${ }^{33}$ A. Gonzalez, R. Denny, and C. Nave, Acta Crystallogr. Sect. D 50, 276 (1994).

${ }^{34}$ C. Emmerich, J. R. Helliwell, M. Redshaw, J. H. Naismith, S. Harrop, J. Raftery, A. J. Kalb (Gilboa), J. Yariv, Z. Dauter, and K. S. Wilson, Acta Crystallogr. Sect. D 50, 749 (1994).

${ }^{35}$ J. H. Naismith, C. Emmerich, J. Habash, S. J. Harrop, J. R. Helliwell, W. N. Hunter, J. Raftery, A. J. Kalb (Gilboa) and J. Yariv, Acta Crystallogr. Sect. D 50, 847 (1994).

${ }^{36}$ M. Tate, E. F. Eikenberry, S. L. Barna, M. E. Wall, J. Lowrence, and S. M. Gruner, J. Appl. Crystallogr. (in press).

${ }^{37}$ S. Ealick and R. L. Walter, Curr. Opinion Struct. Biol. 3, 725 (1993).

${ }^{38}$ T. R. Schneider and K. S. Wilson, Abstr. ACA Annual Meeting Atlanta (1994).

${ }^{30}$ J. R. Helliwell, ESRF "Red Book" (1987).

${ }^{40}$ A. Kvick and A. W. Thompson, ESRF Int. Paper ESRF 23-05, 1992.

${ }^{41}$ C. Wilkinson, Biophys. Chem. (in press).

${ }^{42} \mathrm{~J}$. Morse, in Report of the International Workshop on Area Detectors, Hyogo, Japan, edited by T. Ueki, SPRING-8 Report (1993), pp. 69-79.

${ }^{43}$ S. M. Gruner, S. L. Barna, M. E. Wall, M. W. Tate, and E. F. Eikenberry, Proc. SPIE 2009, 98 (1993).

${ }^{44}$ J. P. Moy, A. Koch, and M. B. Nielsen, Nucl. Instrum. Methods, Vol A 326, 581 (1993).

${ }^{45}$ C. M. Castelli, N. M. Allinson, K. J. Moon, and D. L. Watson, Nucl. Instrum. Methods A 348, 649 (1994).

${ }^{46}$ N. M. Allinson, J. Synchrotron. Radiat. 1, 54 (1994).

${ }^{47}$ E. M. Westbrook, Conceptual Design Report Document No. J9001-2001SA-01, Argonne National Laboratory (1988).

${ }^{48}$ N. M. Allinson, M. Colapietro, J. R. Helliwell, K. Moon, A. W. Thompson, and S. Weisgerber, Rev. Sci. Instrum. 63, 664 (1992). 